\title{
Factor Endocrino en Esterilidad - TIROIDES
}

\author{
Doctor Janne Cortazan \\ Miembro de la Sociadad Colombiana de Endocrinologia \\ Eudocrinólogo del Insti:uto Nacional de Cancerologia.
}

Si se acepta que esterilidad es la inhabilidad para reproducirse, e infertili dad la mavor a menor reduccion de la fertilidad (1), es logico que la esterilidad as la maxima intertilidad posible.

Pedria quiras decirse que esterilidad es la imposibilidad de que un óvulo sear fecundado, y yue inlertilidad es la imposibilidad de que un ovulo fecundado evo lucione hasta un nueve ser viable. Las causas de uno yotro fenomeno son nume. rosas y variadas, y mucho mejor conocidas por ustedes que por quien les habla, como que tales problemas atanen mis al ginecologo obstetra que al endocrinólogo. Sin combargo, un grupo de dichas causas tiene su origen en alteraciones fun comales de la glandula siroides

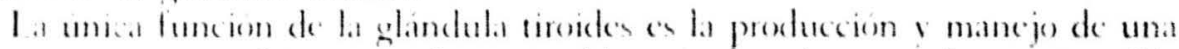
hormen., que en su forme circulante posiblemente es identica a la tiroxina (2).

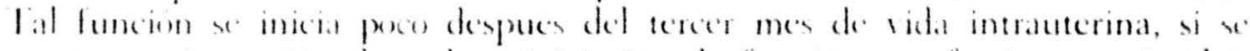
acepta yue captacion de volo e inciacion de funcion on fenomenos simulta neos (3). Desce entonces comicnata la accion de la bormona tiroidiana, y solo terminat totalmente con la mucre.

Del conjunte de complejas acciones de dicha hormonat es conveniente re cordar en enta ocasion tren grupes: 19) en sincrgismo con caracteres genéticos. nutricion. hiprifiso ancrior y gonadas, estintala el crecimiento y desarrolle normales, 2") activa toder las procesos axidaturos posiblemente modificando la fisotogia colular en funcein de cambiex de permeabilidad de membranas celu lanes: 3") dismmene el umbral de irritabilutad det sistema nerviose, y acorta su ticmper de rederis.

I a mancra carcta de accion de la hormona sobre una célula cualquiera es desonecida. pero le investigede hasta ahora hace presumir que actúc como ca talition. 
El organo blanco (target-organ) de la glandula tiroides esta constituide por la totalidad de las celulas que integran un organismo, y asi el ovario y of endometrie tambien están sometidos a su acción general. Ya que la glandula tiroides a su ve er organo blanco de la hipofisis anterior -homoma tirestimu

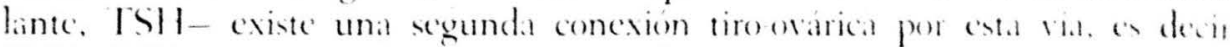
TSH. FSH. LH. La experimentacion en busca de esclarecimente de las inter

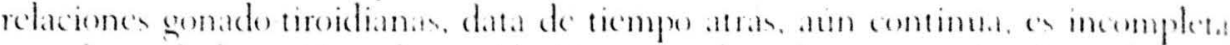

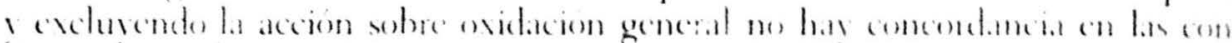

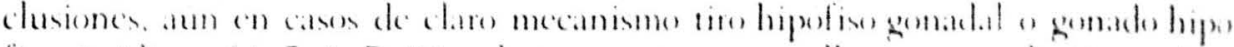

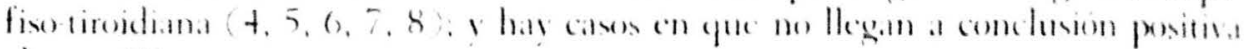
alguna ("),

Resumiendo, la glándula tiroides alecta el ovario y en consecuencia ef endometrio, por tres mecanismos: uno conocido cląramente, la accion general sobre oxidacion: dos mas incompleta o inadesuadamente estudiados: via hipo fisis: via directa diferente a acción general.

La deficiencia de hormona tiroidiana se maniliesta de diversos modos, $x$ gun la edad del individuo $y$, claro esta, segun la magnitud de la deficiencia misma. Si existicta deficiencia total. vale decir ausencia. desde el nacimiento -atireosis, insuficiencia tenal tiroidiana- se tendria, pasado el tiempo. una mujer cretina. Si solamente fucra parcial, o solamente se iniciara en la juventud o en la edad adulta, e tendria un mavor o menor grado de mixedema. En estos gru pos de hipotiroidismo hay hipoplasia genital externa e interna más o menos acentuada, la livide esta disminuida, y el sexappeal ausente. Si se agrega que cl fallo ovulatorio es mus frecuente -aproximadamente $70 \%$ - ('i"), y que has amenorrea o completa anaryuia menstrual en la mavoria de los casos adecuada mente estudiades, en lígico concluir que el hipentroidismo puede ser causi desde infertilidad minima hasta eserilidad. En los coss en referencia la normalidad

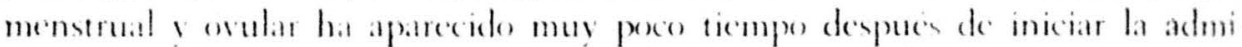
nistacion de turodes y parece que las aleraciones se deban a accion continus de estregenes sobre endometrio, sin oxulacion y en consecuencia sin efecter per nerou de progesteronat

I a cantidad de vexto unido a proteina que se encuentra en la sangre (vede proteinemia aumenta rapidamente en el combaraze a partir de la 3 a semana $y$ baja bruscamente despues del parto ("); el metabolismo basal esta sistemati camente elevade en relacion al normal de la paciente misma, despuée del $2^{\circ}$ mes. Seria mue comveniente que toda mujer antes de expesicion a embaraze. conociera su metaludiomo basal normal, para asi, si no aumenta despué dol $2^{\circ}$ mes de embarase, permitir una firme sospecha de hipotiroidismo.

$S_{e}$ ha comprobado que en hipotirodismo (12-13). La infertilidad por aborto habitual sucede siempre yue la vodoproteinemia no aumenta y por lo contrario permanece en valeres bajes, 2.5 a 3.5 gammas por 100 ece de plasma; que has cambios placentarios que llevan a toxemias (19), y que hay deficit en gonado trofinas. 


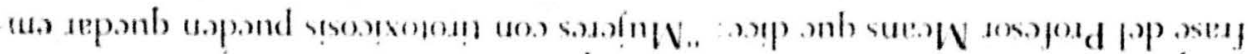

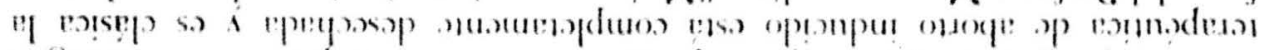

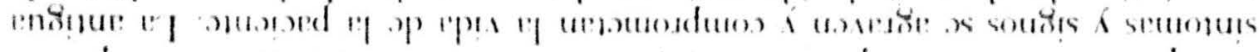

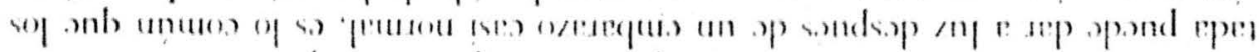

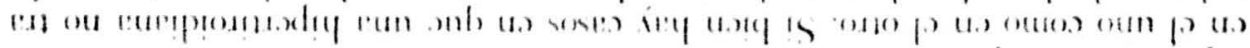

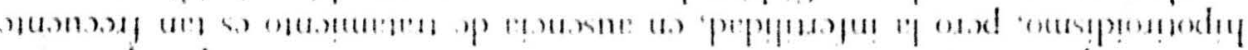

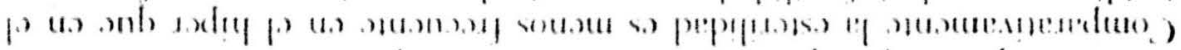

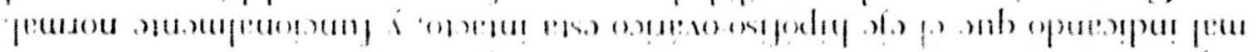

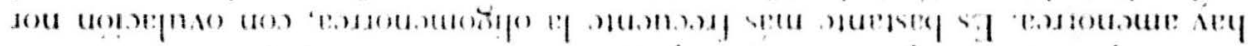

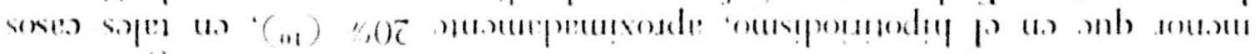

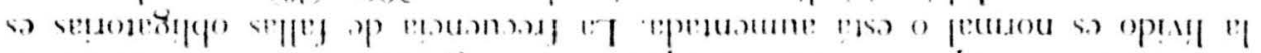

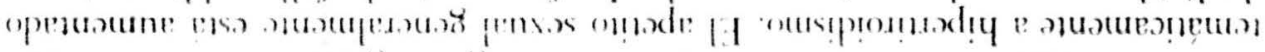

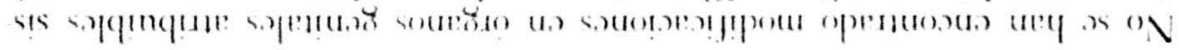

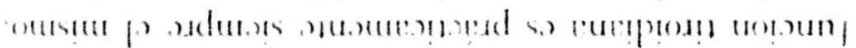

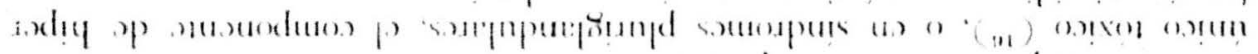

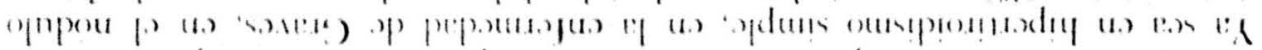

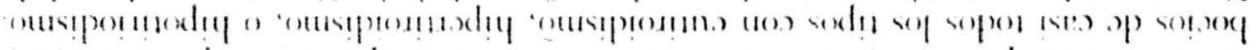

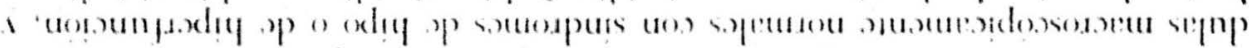
up

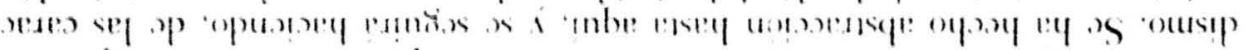

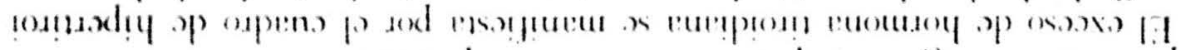

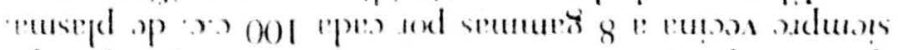

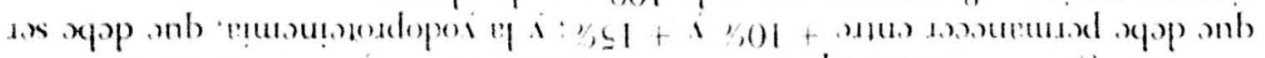

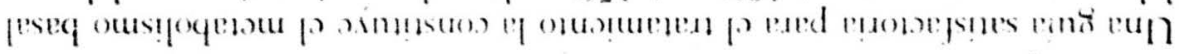

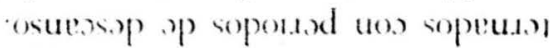

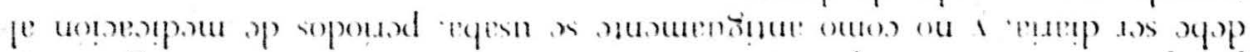

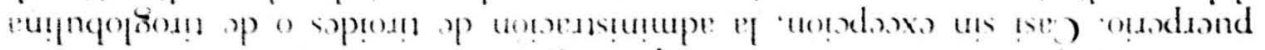

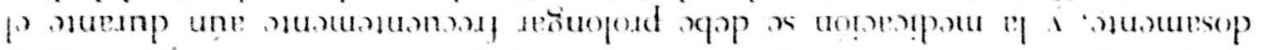

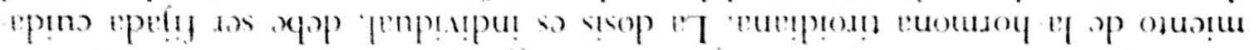

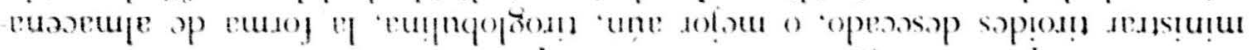

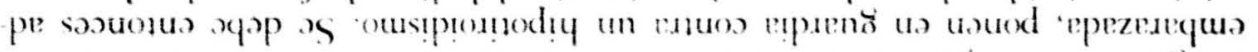

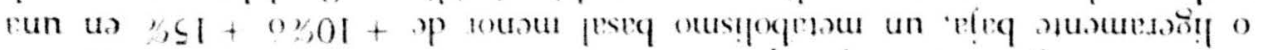

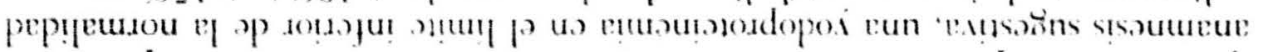

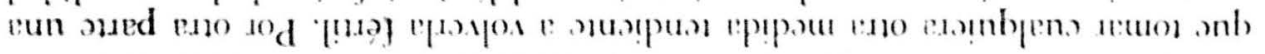

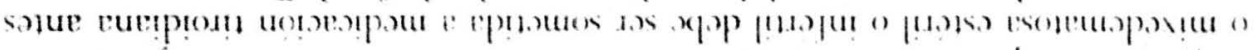

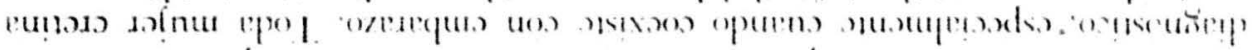

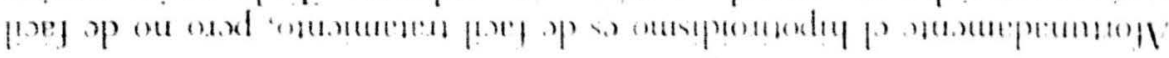

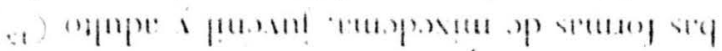

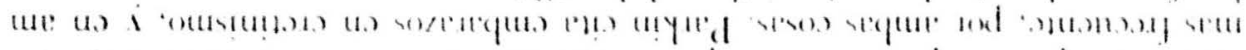
9 '0 '0,

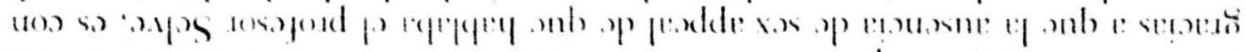

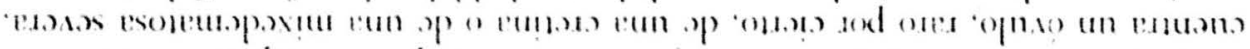

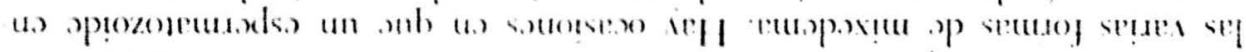
(i.) ! 
barazadas. o mujeres embarazadas pueden volverse tirotoxicósicas. En ambos even. tos es la tirotoxicosis, y no el cmbarazo, lo que debe ser interrumpido" (ii). En ausencia de tratamiento las interrupciones patologicas de embarazo en hiperti roidianas, suceden por aborto o por parto premature. Existen informes de mal fermaciones fetake en partos a termino. y lo más grase de texke, muerte ma ternofetal.

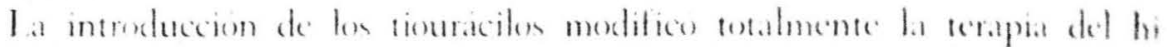

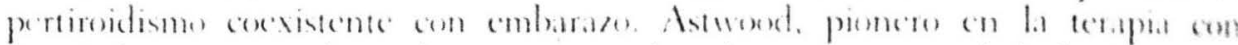

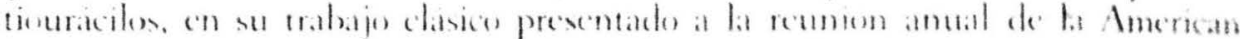
Giviter Assoctation en 1951, csablece claramente la ausencia de complicasos nes maternofetales en at tratamiento de hipertiroidismo mediante tiouracilos en mujeres embrazadas. In 22 embarazos, todos terminaron por parto nomal del nino simo, sin alteraciones de las glandulas tiroides (1n). Parece pues, que si bien los tiourácilos pueden ocasionalmente transponer la barrera placentaria, oho alcanzan a actuar sobre la glandula tiroides fetal, o es excepcional tal trans pesicion.

Actualmente se dispone de + productos principales como antitiroidianos: tres derivados de tiouracilo, y uno del mercaptomidazole: propiltiourácilo, metil. tiouracilo, vodetiouraciles y metilmercaptomidazole. Este último, al peso, es 10 veces mas potente que los tioureaderivados. El yodotiouracilo tiene en combina cion molecular a no en mecela, vodo. y su efecto a más de bloquear la sintess de tiroxina, a manificsta tambien por estacionamiento y aun reduccion del ta mano de la glandula tiroides, gracias a la accion comprobada y de mecanismo des conocide del vodo sobre la producción de TSH.

Recordande que la medida básica en el tratamiento es la administración de uno de los compuestos anteriormente citados, es conveniente, para llevar a ter mino felis un conbaraze ('n hipertiroidiana, administrarle una dieta hipercaloricot particularmente rica en calcos, si no se administra come antitirediano el yode tionracikes suministar vodures a dosis rerapeuticas 2 a 3 gotas de solucion de

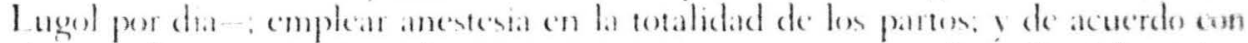
el case clmico suministrate estrogenor, progesterona, o ambos. So dete evitar la alimentacion del hije al pecho ya que entoneces st se le suministraria directa mente antintroidianes en lateche.

Si al combatir un hipertiroidismo el obstetra se excedicra en la dosis de antitiroidianos, correria peligro de pasa de cuadro a hipotiroidismo con peligro evidente para el embarazo. El metabolismo basal adecuadamente determinado, es decir, verdaderamente basal suministra un dato muy valiose para guiar la fera peutica y no debe descender nunca a valores inferiores a $+10 \%$.

Se puede concluer que el status tiroidiano ideal para que hava embarazo not mal, que termine per parto nermal de nuevo individuo normal, debe ser el cati roidismo capar de pasar oportunamente y durante tiempes suficiente, sin intoxi car la paciente, a una hiperfuncion fiscolingica que no es otra cosa que su momo limite superior de normalidad. 


\section{BI B LIOG R A FIA:}

1.-Hurxthal, L. H and Musulin N.: "Clinical Endocrinology". J. B. Lippincott, Philadelphia, Pa., USA. (Pag. 1.099).

2. -Harington, Sir C.: "Twenty-five years of research and biochemistry of thyroid gland". Endocrinology, 49: 401, 1951

3-Chapman, E. M., Corner, G. W. Jr., Robinson, D. and Evans, R. D.: "The collection of radioactive iodine by the human fetal thyroid". J. Clin. Endocri. nol., 8: $717 ; 1948$

4.-Aron, E. et Benoit, J.: "Action antagonisme de la thyreo-stimuline prehypo. physaire et de la folliculine ovarienne sur le functionamient thyroidien". Comp. Rend. Soc. de Biol. 109: 923; 1932.

5.-Chu, J. P. and You, S. S.: "The role of thyroid gland and oestrogen in the regulation of gonadothopic activity of the anterior pituitary". J. Clin. Endocrinol., 4: 115; 1945.

6.-Korenchevsky, V.: "Influence of the chryptorchidism and of castration on body weight, fat deposition, sexual and endocrine organs of male rat". J. Path. and Bact., 33: 607; 1930.

7.-Kippen, A. A. and Loeb, L.: "Effect of gonadectomy on thyroid gland in ginea pig". Endocrinology, 20: 201; 1936.

8. - Cohen, C. S.: "Effect of experimentally produced hyperthyroidism upon reproductive and associated organs of male rats". Amer. J. Anat., 56: 143; 1935.

9.-Kunde, M. M., D'Amour, F. F., Carlson, A. J. and Gustavson, R. G.: "Studies on metabolism. VIII The effect of estrin injections on the basal metabolismo. uterine endometrium, lactatio, mating and maternal instincts in the adult dog". Am. J. Physiol., 95: 630; 1930.

10.-Goldsmith, R. E., Sturgis, S. H., Lerman, J. and Stambury, J. B.: "The menstrual pattern in throid disease". J. Clin. Endocrinol., 12: 946; 1952.

11.-Rapport, R. L. and Curtis, G. M.: "The clinical significance of blood jodine: a review". J. Clin. Endocrinol., 10: 735; 1950

12. Heinemann, M., Johnson, C. E. and Man, E. B.: "Serum precipitable ioidine con. centrations in pregnancy". J. Clin. Invest., 27: 91; 1948 .

13. - Peters, J. P., Man, E. B. and Heinemann, M.: "Pregnancy and thyroid gland". Yale J. Biol. and Med., 20: 449; 1948.

14 -Soberon, G. y Rodriguez, R.: "Hipertiroidismo y embarazo", Rev. Invest Chn. (Mexico), 4: 19; 1952

15. - Parkin, G. and Greene, A.: "Pregnancy occurring $1 n$ cretinism, and in juve nile and adult myxedema". J Clin. Endocrirol., 3: 466; 1943

16. Cope, O., Rawson, R. W. and McArthur, J. W.: "The hyperfunctioning single adenoma of the thyroid". Surg. Gynec. \& Obst., 84: 415; 1947.

17. -Menas, J. H.: "The Thyroid and ins diseases". 2nd ed. J. B. Lippincott, Philadelphia, Pa., USA. (Pag. 437).

18. - Astwood. E. B.: "The use of antithyroid drugs during pregnancy". J. Clin. endocrinol., 11: 1.045: 1951 . 